\title{
ГАРМОНИЧЕСКИЕ ИНТЕРПОЛЯЦИОННЫЕ ВСПЛЕСКИ В КРАЕВОЙ ЗАДАЧЕ НЕЙМАНА В КОЛЬЦЕ
}

\author{
Д. А. Ямковой
}

\begin{abstract}
В данной статье рассматривается краевая задача Неймана в центрально-симметричном кольце с единичным внешним радиусом и непрерывными граничными значениями. Решение поставленной задачи основано на разложении в ряд непрерывных граничных значений по интерполяционным и интерполяционноортогональным $2 \pi$-периодическим всплескам, состоящим из тригонометрических полиномов. Идея подобного разложения и конструкция интерполяционных и интерполяционно-ортогональных $2 \pi$-периодических всплесков, построенных на основе функций мейеровского типа, принадлежат Ю. Н. Субботину и Н. И. Черных. Удобство построенных рядов состоит в том, что они легко продолжаются до гармонических в круге полиномов, с помощью которых уже удается представить решение исходной задачи в кольце в виде двух равномерно сходящихся в замыкании этого кольца рядов. Также коэффициенты этих рядов легко считаются и не требуют вычисления интегралов. В результате получено точное представление решения краевой задачи Неймана в кольце в виде двух рядов по упомянутой выше системе гармонических всплесков, и найдена погрешность приближения точного решения частичными суммами этих рядов.
\end{abstract}

Ключевые слова: интерполяционные всплески, гармонические функции, задача Неймана.

D. A. Yamkovoi. Harmonic interpolating wavelets in the Neumann boundary value problem in a ring.

We consider the Neumann boundary value problem with continuous boundary values in a centrally symmetric ring with unit outer radius. The approach to solving the problem is based on expanding the continuous boundary values in interpolating and interpolating orthogonal $2 \pi$-periodic wavelets consisting of trigonometric polynomials. The idea for such an expansion and the scheme of interpolating and interpolating orthogonal $2 \pi$-periodic wavelets based on Meyer-type wavelets were proposed by Yu.N. Subbotin and N.I. Chernykh. It is convenient to use these series due to the fact that they are easily extended to polynomials harmonic in a circle, and the harmonic polynomials can be used to present the solution of the original problem in a ring as two series uniformly convergent in the closure of the ring. Moreover, the coefficients of the series are easily calculated and do not require the calculation of integrals. As a result, we obtain an exact representation for the solution of the Neumann boundary value problem in the ring in the form of two series in the mentioned system of harmonic wavelets and find an estimate for the error of approximating the exact solution by partial sums of the series.

Keywords: interpolating wavelets, harmonic functions, Neumann boundary value problem.

MSC: 35J25, 41A05

DOI: $10.21538 / 0134-4889-2020-26-4-279-289$

\section{Введение}

В работе [1] Ю. Н. Субботин и Н. И. Черных используют вещественные ортогональные $2 \pi$ периодические всплески, построенные ими на основе всплесков из работ [2;3], для решения краевых задач типа Дирихле, Неймана и Пуассона в круге и кольце для гармонических и бигармонических функций. Продолжая свои предыдущие работы, Ю. Н. Субботин и Н. И. Черных в статье [4] применяют уже интерполяционные и интерполяционно-ортогональные $2 \pi$-периодические всплески из их совместной работы [5] для решения краевой задачи Дирихле в круге. Эти же идеи были использованы Ю. Н. Субботиным и Н. И. Черных в работе 2018 г. ( Тp. Ин-та математики и механики УрО РАН. 2018. Т. 24, № 4. С. 225-234) для решения краевой задачи Дирихле в центрально-симметричном кольце и автором статьи в [6] для решения краевой задачи Неймана в круге.

\footnotetext{
${ }^{1}$ Работа выполнена в рамках исследований, проводимых в Уральском математическом центре.
} 
В этой статье интерполяционные и интерполяционно-ортогональные $2 \pi$-периодические системы базисов всплесков из работы [5] применяются для построения гармонических в центрально-симметричном кольце с единичным внешним радиусом всплесков и решения краевой задачи Неймана в данном кольце. Нас интересует точное представление решения задачи в виде двух равномерно сходящихся в замыкании кольца рядов по упомянутой выше системе базисов всплесков, а также поведение частичных сумм и погрешность приближения данными суммами точного решения.

\section{1. Основные обозначения}

Как в работах [4;5], в качестве $\widehat{\varphi}(\omega)$ будем брать преобразование Фурье вещественнозначной функции типа Мейера (см. $[2 ; 3]) \widehat{\varphi}_{\varepsilon}(\omega)$

$$
\begin{cases}\widehat{\varphi}_{\varepsilon}(\omega)=0, & |\omega|>(1+\varepsilon) / 2 \\ \widehat{\varphi}_{\varepsilon}(\omega)=1, & |\omega| \leq(1-\varepsilon) / 2 \\ \widehat{\varphi}_{\varepsilon}^{2}(\omega)+\widehat{\varphi}_{\varepsilon}^{2}(\omega-1)=1, & (1-\varepsilon) / 2<\omega<(1+\varepsilon) / 2,\end{cases}
$$

где $\varepsilon \in(0,1 / 3]$. Также потребуем от функции $\widehat{\varphi}_{\varepsilon}^{2}(\omega)$, чтобы она была четной, гладкой, с центром симметрии над промежутком $((1-\varepsilon) / 2,(1+\varepsilon) / 2)$ в точке $1 / 2$. В качестве функции $\widehat{\varphi}_{\varepsilon}(\omega)$, удовлетворяющей указанным выше условиям, подойдет, например, функция

$$
\widehat{\varphi}_{\varepsilon}^{2}(\omega):= \begin{cases}0, & |\omega| \geq(1+\varepsilon) / 2 \\ 1, & |\omega| \leq(1-\varepsilon) / 2 \\ \cos ^{2}\left(\pi \frac{\omega-(1-\varepsilon) / 2}{2 \varepsilon}\right), & (1-\varepsilon) / 2<|\omega|<(1+\varepsilon) / 2 .\end{cases}
$$

На основе заданной так функции $\widehat{\varphi}_{\varepsilon}(\omega)$ строятся интерполяционно-ортогональные $2 \pi$-периодические всплески

$$
\Phi_{s}^{j, k}(x)=2^{-j} \sum_{\nu \in \Delta_{\varepsilon}^{j} \cap \mathbb{Z}} \widehat{\varphi}_{s}\left(\frac{\nu}{2^{j}}\right) e^{i \nu\left(x-2 \pi k / 2^{j}\right)}, \quad s=1,2,3, \quad j \in \mathbb{N} \cup\{0\}, \quad k=\overline{0,2^{j}-1},
$$

где $\Delta_{\varepsilon}^{j}=\left(-2^{j-1}(1+\varepsilon), 2^{j-1}(1+\varepsilon)\right)$,

$$
\widehat{\varphi}_{2}(\omega)=\widehat{\varphi}_{\varepsilon}^{2}(\omega)+i \operatorname{sign}(\omega) \beta(\omega), \quad \beta(\omega)=\widehat{\varphi}_{\varepsilon}(\omega)\left(\widehat{\varphi}_{\varepsilon}(\omega-1)+\widehat{\varphi}_{\varepsilon}(\omega+1)\right), \quad \widehat{\varphi}_{3}(\omega)=\widehat{\varphi}_{\varepsilon}^{2}(\omega),
$$

а формулу для более громоздкого $\widehat{\varphi}_{1}(\omega)$ можно найти в [4]. При $s=3$ получаем интерполяционные в $C_{2 \pi}$ (пространство непрерывных $2 \pi$-периодических функций) на сетках $\left\{x_{j}^{k}:=\right.$ $\left.2 \pi k / 2^{j}: k=0, \ldots, 2^{j}-1\right\}$ всплески, а при $s=1,2$ - интерполяционные в $C_{2 \pi}$ на тех же сетках и одновременно ортогональные в $L^{2}[0,2 \pi]$ всплески.

Далее все выкладки, где фигурирует $s$, проводятся для $s=1,2,3$.

Из работы [6] следует, что функция $f \in C_{2 \pi}$ представима в виде равномерно сходящегося на $\mathbb{R}$ ряда по $\Phi_{s}^{j+1,2 k+1}(x)$, т. е.

$$
f(x)=f(0)+\sum_{j=0}^{+\infty} \sum_{k=0}^{2^{j}-1}\left(f\left(x_{j+1}^{2 k+1}\right)-S_{s, 2^{j}}\left(x_{j+1}^{2 k+1} ; f\right)\right) \Phi_{s}^{j+1,2 k+1}(x),
$$

где

$$
S_{s, 2^{j}}(x ; f)=\sum_{k=0}^{2^{j}-1} f\left(\frac{2 \pi k}{2^{j}}\right) \Phi_{s}^{j, k}(x), \quad j \in \mathbb{N} \cup\{0\},
$$


есть интерполяционная проекция функции $f$ на подпространство $V_{s}^{j}=\operatorname{span}\left\{\Phi_{s}^{j, k}(x): k=\right.$ $\left.\overline{0,2^{j}-1}\right\}: j \in \mathbb{Z}$ (см. [4]). Для дальнейшего удобства обозначим

$$
c_{j, k}(f):=f\left(x_{j+1}^{2 k+1}\right)-S_{s, 2^{j}}\left(x_{j+1}^{2 k+1} ; f\right), \quad j \in \mathbb{N} \cup\{0\}, \quad k=\overline{0,2^{j}-1} .
$$

Всплески $\Phi_{s}^{j, k}(x)$ - есть тригонометрические полиномы по системе $\left\{e^{i \nu x}: \nu \in \Delta_{\varepsilon}^{j} \cap \mathbb{Z}\right\}$, которые легко продолжаются до гармонических в круге $K_{1}:=\left\{z=r e^{i x}: r<1,0 \leq x<2 \pi\right\}$ полиномов добавлением множителя $r^{|\nu|}$ :

$$
\Phi_{s}^{j, k}(r, x):=2^{-j} \sum_{\nu \in \Delta_{\varepsilon}^{j} \cap \mathbb{Z}} \widehat{\varphi}_{s}\left(\frac{\nu}{2^{j}}\right) r^{|\nu|} e^{i \nu\left(x-2 \pi k / 2^{j}\right)}, \quad j \in \mathbb{N} \cup\{0\}, \quad k=\overline{0,2^{j}-1},
$$

и гармонических вне замыкания круга $K_{\rho}:=\left\{z=r e^{i x}: r<\rho, 0 \leq x<2 \pi\right\}$ радиуса $\rho$ полиномов добавлением множителя $(\rho / r)^{|\nu|}$ :

$$
\Phi_{s}^{j, k}\left(\frac{\rho}{r}, x\right):=2^{-j} \sum_{\nu \in \Delta_{\varepsilon}^{j} \cap \mathbb{Z}} \widehat{\varphi}_{s}\left(\frac{\nu}{2^{j}}\right)\left(\frac{\rho}{r}\right)^{|\nu|} e^{i \nu\left(x-2 \pi k / 2^{j}\right)}, \quad j \in \mathbb{N} \cup\{0\}, \quad k=\overline{0,2^{j}-1} .
$$

\section{2. Решение задачи Неймана в кольце с помощью гармонических интерполяционных всплесков}

Рассмотрим постановку задачи Неймана в кольце $R_{\rho}:=\left\{z=r e^{i x}: 0<\rho<r<1,0 \leq x<\right.$ $2 \pi\}$ в полярной системе координат:

$$
\left\{\begin{array}{l}
\Delta U(r, x)=0, \quad U(r, x) \in C^{1}\left(\overline{R_{\rho}}\right) \cap C^{2}\left(R_{\rho}\right), \\
\partial U(1, x) / \partial r=g_{1}(x) \in C_{2 \pi} \\
-\partial U(\rho, x) / \partial r=g_{\rho}(x) \in C_{2 \pi} .
\end{array}\right.
$$

Хорошо известно, что необходимым условием разрешимости задачи (2.1) является условие

$$
\int_{0}^{2 \pi}\left(g_{1}(x)+\rho g_{\rho}(x)\right) d x=0 .
$$

Так как функции $g_{1}, g_{\rho}$ - из пространства $C_{2 \pi}$, то, как было отмечено ранее, их можно представить в виде равномерно сходящихся рядов

$$
\begin{aligned}
& g_{1}(x)=g_{1}(0)+\sum_{j=0}^{+\infty} \sum_{k=0}^{2^{j}-1} c_{j, k}\left(g_{1}\right) \Phi_{s}^{j+1,2 k+1}(x), \\
& g_{\rho}(x)=g_{\rho}(0)+\sum_{j=0}^{+\infty} \sum_{k=0}^{2^{j}-1} c_{j, k}\left(g_{\rho}\right) \Phi_{s}^{j+1,2 k+1}(x) .
\end{aligned}
$$

Теорема 1. В условиях, сформулированных при постановке задачи (2.1), при каждом $s=1,2,3$ справедливо представление для точного решения задачи (2.1)

$$
\begin{aligned}
& U(r, x)=\sum_{j=0}^{+\infty} \sum_{k=0}^{2^{j}-1} 2^{-(j+1)} \sum_{\nu \in \Delta_{\varepsilon}^{j+1} \cap \mathbb{Z} \backslash\{0\}} c_{j, k}\left(g_{1}(\cdot)\right) \widehat{\varphi}_{s}\left(\frac{\nu}{2^{j+1}}\right) \frac{r^{2|\nu|}+\rho^{2|\nu|}}{|\nu| r^{|\nu|}\left(1-\rho^{2|\nu|}\right)} e^{i \nu\left(x-2 \pi(2 k+1) / 2^{j+1}\right)} \\
& +\sum_{j=0}^{+\infty} \sum_{k=0}^{2^{j}-1} 2^{-(j+1)} \sum_{\nu \in \Delta_{\varepsilon}^{j+1} \cap \mathbb{Z} \backslash\{0\}} c_{j, k}\left(g_{\rho}(\cdot)\right) \widehat{\varphi}_{s}\left(\frac{\nu}{2^{j+1}}\right) \frac{\rho^{|\nu|+1}\left(r^{2|\nu|}+1\right)}{|\nu| r^{|\nu|}\left(1-\rho^{2|\nu|}\right)} e^{i \nu\left(x-2 \pi(2 k+1) / 2^{j+1}\right)}
\end{aligned}
$$




$$
+\left(g_{1}(0)+\sum_{j=0}^{+\infty} \sum_{k=0}^{2^{j}-1} c_{j, k}\left(g_{1}(\cdot)\right) 2^{-(j+1)}\right) \ln r+U_{0}, \quad(r, x): z=r e^{i x} \in \overline{R_{\rho}} .
$$

Здесъ $U_{0}-$ произвольная вещественная константа, коэффициенты $c_{j, k}\left(g_{1}(\cdot)\right) ; c_{j, k}\left(g_{\rho}(\cdot)\right)$ находятся по формулам (1.2), (1.3), а оба ряда в правой части (2.4) сходятся равномерно в $\overline{R_{\rho}}$.

Д о к а з а т е л ь с т в о. Из [7, с. 261] следует, что решение задачи (2.1) существует, единственно с точностью до аддитивной константы $U_{0}$ и представимо в виде

$$
U(r, x)=U_{1}(r, x)+U_{\rho}(r, x)+A \ln r+U_{0}, \quad(r, x): z=r e^{i x} \in \overline{R_{\rho}} .
$$

Здесь $U_{1}(r, x)$ - гармоническая в $K_{1}$ и непрерывно-дифференцируемая в $\overline{K_{1}}$ функция; $U_{\rho}(r, x)$ - гармоническая вне $\overline{K_{\rho}}$ и непрерывно-дифференцируемая вне $K_{\rho}$ функция, причем $U_{\rho}(\infty)=\lim _{z \rightarrow \infty} U_{\rho}(z)=0$, а $A, U_{0}$ - вещественные константы. Продифференцируем по $r$ представление (2.5) и подставим его в граничные условия из (2.1). Равенство для второго граничного условия умножим на $\rho$. Получим систему

$$
\left\{\begin{array}{l}
\partial U_{1}(1, x) / \partial r+\partial U \rho(1, x) / \partial r+A=g_{1}(x), \\
-\rho \partial U_{1}(\rho, x) / \partial r-\rho \partial U_{\rho}(\rho, x) / \partial r-A=\rho g_{\rho}(x) .
\end{array}\right.
$$

Так как функции $r \partial U_{1}(r, x) / \partial r$ и $r \partial U_{\rho}(r, x) / \partial r$ гармонические в $K_{1}$ и вне $\overline{K_{\rho}}$ соответственно и непрерывные в $\overline{K_{1}}$ и вне $K_{\rho}$ соответственно, то из доказательства [6, теоремы 1$]$ следует, что они представимы в виде равномерно сходящихся в $\overline{K_{1}}$ и вне $K_{\rho}$ рядов по гармоническим в $K_{1}$ и вне $\overline{K_{\rho}}$ полиномам $\Phi_{s}^{j+1,2 k+1}(r, x)$ и $\Phi_{s}^{j+1,2 k+1}(\rho / r, x)$ соответственно

$$
\begin{gathered}
r \frac{\partial U_{1}}{\partial r}(r, x)=\frac{\partial U_{1}}{\partial r}(1,0)+\sum_{j=0}^{+\infty} \sum_{k=0}^{2^{j}-1} c_{j, k}\left(\frac{\partial U_{1}}{\partial r}(1, \cdot)\right) \Phi_{s}^{j+1,2 k+1}(r, x), \quad(r, x): z=r e^{i x} \in \overline{K_{1}}, \\
r \frac{\partial U_{\rho}}{\partial r}(r, x)=\rho \frac{\partial U_{\rho}}{\partial r}(\rho, 0)+\sum_{j=0}^{+\infty} \sum_{k=0}^{2^{j}-1} c_{j, k}\left(\rho \frac{\partial U_{\rho}}{\partial r}(\rho, \cdot)\right) \Phi_{s}^{j+1,2 k+1}\left(\frac{\rho}{r}, x\right), \\
(r, x): z=r e^{i x} \in \mathbb{C} \backslash K_{\rho},
\end{gathered}
$$

где $c_{j, k}\left(\partial U_{1}(1, \cdot) / \partial r\right), c_{j, k}\left(\rho \partial U_{\rho}(\rho, \cdot) / \partial r\right)$ - неизвестные коэффициенты.

Используя формулы (1.1), (1.4) и (1.5), подставим представления (2.3) и (2.7) в систему (2.6) и получим следующую систему из двух уравнений:

$$
\begin{aligned}
\frac{\partial U_{1}}{\partial r}(1,0)+ & \sum_{j=0}^{+\infty} \sum_{k=0}^{2^{j}-1} c_{j, k}\left(\frac{\partial U_{1}}{\partial r}(1, \cdot)\right) 2^{-(j+1)} \sum_{\nu \in \Delta_{\varepsilon}^{j+1} \cap \mathbb{Z}} \widehat{\varphi}_{s}\left(\frac{\nu}{2^{j+1}}\right) e^{i \nu\left(x-2 \pi(2 k+1) / 2^{j+1}\right)}+\rho \frac{\partial U_{\rho}}{\partial r}(\rho, 0) \\
& +\sum_{j=0}^{+\infty} \sum_{k=0}^{2^{j}-1} c_{j, k}\left(\rho \frac{\partial U_{\rho}}{\partial r}(\rho, \cdot)\right) 2^{-(j+1)} \sum_{\nu \in \Delta_{\varepsilon}^{j+1} \cap \mathbb{Z}} \widehat{\varphi}_{s}\left(\frac{\nu}{2^{j+1}}\right) \rho^{|\nu|} e^{i \nu\left(x-2 \pi(2 k+1) / 2^{j+1}\right)}+A \\
= & g_{1}(0)+\sum_{j=0}^{+\infty} \sum_{k=0}^{2^{j}-1} c_{j, k}\left(g_{1}(\cdot)\right) 2^{-(j+1)} \sum_{\nu \in \Delta_{\varepsilon}^{j+1} \cap \mathbb{Z}} \widehat{\varphi}_{s}\left(\frac{\nu}{2^{j+1}}\right) e^{i \nu\left(x-2 \pi(2 k+1) / 2^{j+1}\right)}, \\
-\frac{\partial U_{1}}{\partial r}(1,0)- & \sum_{j=0}^{+\infty} \sum_{k=0}^{2^{j}-1} c_{j, k}\left(\frac{\partial U_{1}}{\partial r}(1, \cdot)\right) 2^{-(j+1)} \sum_{\nu \in \Delta_{\varepsilon}^{j+1} \cap \mathbb{Z}} \widehat{\varphi}_{s}\left(\frac{\nu}{2^{j+1}}\right) \rho^{|\nu|} e^{i \nu\left(x-2 \pi(2 k+1) / 2^{j+1}\right)}-\rho \frac{\partial U_{\rho}}{\partial r}(\rho, 0) \\
& -\sum_{j=0}^{+\infty} \sum_{k=0}^{2^{j}-1} c_{j, k}\left(\rho \frac{\partial U_{\rho}}{\partial r}(\rho, \cdot)\right) 2^{-(j+1)} \sum_{\nu \in \Delta_{\varepsilon}^{j+1} \cap \mathbb{Z}} \widehat{\varphi}_{s}\left(\frac{\nu}{2^{j+1}}\right) e^{i \nu\left(x-2 \pi(2 k+1) / 2^{j+1}\right)}-A \\
= & \rho g_{\rho}(0)+\rho \sum_{j=0}^{+\infty} \sum_{k=0}^{2^{j}-1} c_{j, k}\left(g_{\rho}(\cdot)\right) 2^{-(j+1)} \sum_{\nu \in \Delta_{\varepsilon}^{j+1} \cap \mathbb{Z}} \widehat{\varphi}_{s}\left(\frac{\nu}{2^{j+1}}\right) e^{i \nu\left(x-2 \pi(2 k+1) / 2^{j+1}\right)} .
\end{aligned}
$$


Мы можем поменять порядок суммирования в рядах в полученной системе из двух уравнений, так как данные ряды сходятся равномерно. Поменяем порядок суммирования по $j$ и $\nu$ и с учетом равенства $\widehat{\varphi}_{s}(0)=1$ получим систему из уравнений $(2.8),(2.9)$

$$
\begin{aligned}
& \frac{\partial U_{1}}{\partial r}(1,0)+\sum_{j=0}^{+\infty} \sum_{k=0}^{2^{j}-1} c_{j, k}\left(\frac{\partial U_{1}}{\partial r}(1, \cdot)\right) 2^{-(j+1)} \\
& +\sum_{\nu \in \mathbb{Z} \backslash\{0\}}\left[\sum_{j=\nabla_{\varepsilon}^{|\nu|}}^{+\infty} \sum_{k=0}^{2^{j}-1} c_{j, k}\left(\frac{\partial U_{1}}{\partial r}(1, \cdot)\right) 2^{-(j+1)} \widehat{\varphi}_{s}\left(\frac{\nu}{2^{j+1}}\right) e^{-2 \pi i \nu(2 k+1) / 2^{j+1}}\right] e^{i \nu x} \\
& +\rho \frac{\partial U_{\rho}}{\partial r}(\rho, 0)+\sum_{j=0}^{+\infty} \sum_{k=0}^{2^{j}-1} c_{j, k}\left(\rho \frac{\partial U_{\rho}}{\partial r}(\rho, \cdot)\right) 2^{-(j+1)} \\
& +\sum_{\nu \in \mathbb{Z} \backslash\{0\}}\left[\sum_{j=\nabla_{\varepsilon}^{|\nu|}}^{+\infty} \sum_{k=0}^{2^{j}-1} c_{j, k}\left(\rho \frac{\partial U_{\rho}}{\partial r}(\rho, \cdot)\right) 2^{-(j+1)} \widehat{\varphi}_{s}\left(\frac{\nu}{2^{j+1}}\right) e^{-2 \pi i \nu(2 k+1) / 2^{j+1}}\right] \rho^{|\nu|} e^{i \nu x}+A \\
& =g_{1}(0)+\sum_{j=0}^{+\infty} \sum_{k=0}^{2^{j}-1} c_{j, k}\left(g_{1}(\cdot)\right) 2^{-(j+1)} \\
& +\sum_{\nu \in \mathbb{Z} \backslash\{0\}}\left[\sum_{j=\nabla_{\varepsilon}^{|\nu|}}^{+\infty} \sum_{k=0}^{2^{j}-1} c_{j, k}\left(g_{1}(\cdot)\right) 2^{-(j+1)} \widehat{\varphi}_{s}\left(\frac{\nu}{2^{j+1}}\right) e^{-2 \pi i \nu(2 k+1) / 2^{j+1}}\right] e^{i \nu x}, \\
& -\frac{\partial U_{1}}{\partial r}(1,0)-\sum_{j=0}^{+\infty} \sum_{k=0}^{2^{j}-1} c_{j, k}\left(\frac{\partial U_{1}}{\partial r}(1, \cdot)\right) 2^{-(j+1)} \\
& -\sum_{\nu \in \mathbb{Z} \backslash\{0\}}\left[\sum_{j=\nabla_{\varepsilon}^{|\nu|}}^{+\infty} \sum_{k=0}^{2^{j}-1} c_{j, k}\left(\frac{\partial U_{1}}{\partial r}(1, \cdot)\right) 2^{-(j+1)} \widehat{\varphi}_{s}\left(\frac{\nu}{2^{j+1}}\right) e^{-2 \pi i \nu(2 k+1) / 2^{j+1}}\right] \rho^{|\nu|} e^{i \nu x} \\
& -\rho \frac{\partial U_{\rho}}{\partial r}(\rho, 0)-\sum_{j=0}^{+\infty} \sum_{k=0}^{2^{j}-1} c_{j, k}\left(\rho \frac{\partial U_{\rho}}{\partial r}(\rho, \cdot)\right) 2^{-(j+1)} \\
& -\sum_{\nu \in \mathbb{Z} \backslash\{0\}}\left[\sum_{j=\nabla_{\varepsilon}^{|\nu|}}^{+\infty} \sum_{k=0}^{2^{j}-1} c_{j, k}\left(\rho \frac{\partial U_{\rho}}{\partial r}(\rho, \cdot)\right) 2^{-(j+1)} \widehat{\varphi}_{s}\left(\frac{\nu}{2^{j+1}}\right) e^{-2 \pi i \nu(2 k+1) / 2^{j+1}}\right] e^{i \nu x}-A \\
& =\rho g_{\rho}(0)+\rho \sum_{j=0}^{+\infty} \sum_{k=0}^{2^{j}-1} c_{j, k}\left(g_{\rho}(\cdot)\right) 2^{-(j+1)} \\
& +\rho \sum_{\nu \in \mathbb{Z} \backslash\{0\}}\left[\sum_{j=\nabla_{\varepsilon}^{|\nu|}}^{+\infty} \sum_{k=0}^{2^{j}-1} c_{j, k}\left(g_{\rho}(\cdot)\right) 2^{-(j+1)} \times \widehat{\varphi}_{s}\left(\frac{\nu}{2^{j+1}}\right) e^{-2 \pi i \nu(2 k+1) / 2^{j+1}}\right] e^{i \nu x},
\end{aligned}
$$

где

$$
\nabla_{\varepsilon}^{|\nu|}= \begin{cases}{\left[\log _{2}\left(\frac{|\nu|}{1+\varepsilon}\right)\right],} & \log _{2}\left(\frac{|\nu|}{1+\varepsilon}\right) \notin \mathbb{Z}, \\ \log _{2}\left(\frac{|\nu|}{1+\varepsilon}\right)+1, & \log _{2}\left(\frac{|\nu|}{1+\varepsilon}\right) \in \mathbb{Z} .\end{cases}
$$

В системе (2.8), (2.9) множители при $e^{i \nu x}-$ это коэффициенты Фурье непрерывных $2 \pi-$ периодических функций $\partial U_{1}(1, x) / \partial r, \rho \partial U_{\rho}(\rho, x) / \partial r, g_{1}(x)$ и $g_{\rho}(x)$, поэтому для того чтобы 
выполнялись оба равенства, коэффициенты при $e^{i \nu x}$ в обеих частях обоих равенств системы должны совпадать.

Сравним свободные коэффициенты (при $\nu=0$ ) в системе $(2.8),(2.9)$ и получим систему

$$
\left\{\begin{array}{l}
\frac{\partial U_{1}}{\partial r}(1,0)+\sum_{j=0}^{+\infty} \sum_{k=0}^{2^{j}-1} c_{j, k}\left(\frac{\partial U_{1}}{\partial r}(1, \cdot)\right) 2^{-(j+1)}+\rho \frac{\partial U_{\rho}}{\partial r}(\rho, 0) \\
+\sum_{j=0}^{+\infty} \sum_{k=0}^{2^{j}-1} c_{j, k}\left(\rho \frac{\partial U_{\rho}}{\partial r}(\rho, \cdot)\right) 2^{-(j+1)}+A=g_{1}(0)+\sum_{j=0}^{+\infty} \sum_{k=0}^{2^{j}-1} c_{j, k}\left(g_{1}(\cdot)\right) 2^{-(j+1)}, \\
-\frac{\partial U_{1}}{\partial r}(1,0)-\sum_{j=0}^{+\infty} \sum_{k=0}^{2^{j}-1} c_{j, k}\left(\frac{\partial U_{1}}{\partial r}(1, \cdot)\right) 2^{-(j+1)}-\rho \frac{\partial U_{\rho}}{\partial r}(\rho, 0) \\
-\sum_{j=0}^{+\infty} \sum_{k=0}^{2^{j}-1} c_{j, k}\left(\rho \frac{\partial U_{\rho}}{\partial r}(\rho, \cdot)\right) 2^{-(j+1)}-A=\rho g_{\rho}(0)+\rho \sum_{j=0}^{+\infty} \sum_{k=0}^{2^{j}-1} c_{j, k}\left(g_{\rho}(\cdot)\right) 2^{-(j+1)}
\end{array} .\right.
$$

Перепишем условие (2.2), подставив в него формулы из (2.3):

$$
0=\int_{0}^{2 \pi}\left(g_{1}(x)+\rho g_{\rho}(x)\right) d x=2 \pi\left(g_{1}(0)+\rho g_{\rho}(0)+\sum_{j=0}^{+\infty} \sum_{k=0}^{2^{j}-1}\left(c_{j, k}\left(g_{1}(\cdot)\right)+\rho c_{j, k}\left(g_{\rho}(\cdot)\right)\right) 2^{-(j+1)}\right),
$$

т. е. получим, что

$$
-\rho g_{\rho}(0)-\rho \sum_{j=0}^{+\infty} \sum_{k=0}^{2^{j}-1} c_{j, k}\left(g_{\rho}(\cdot)\right) 2^{-(j+1)}=g_{1}(0)+\sum_{j=0}^{+\infty} \sum_{k=0}^{2^{j}-1} c_{j, k}\left(g_{1}(\cdot)\right) 2^{-(j+1)} .
$$

В системе (2.10) вычтем из первого уравнения второе:

$$
\begin{gathered}
A=-\frac{\partial U_{1}}{\partial r}(1,0)-\sum_{j=0}^{+\infty} \sum_{k=0}^{2^{j}-1} c_{j, k}\left(\frac{\partial U_{1}}{\partial r}(1, \cdot)\right) 2^{-(j+1)} \\
-\rho \frac{\partial U_{\rho}}{\partial r}(\rho, 0)-\sum_{j=0}^{+\infty} \sum_{k=0}^{2^{j}-1} c_{j, k}\left(\rho \frac{\partial U_{\rho}}{\partial r}(\rho, \cdot)\right) 2^{-(j+1)}+g_{1}(0)+\sum_{j=0}^{+\infty} \sum_{k=0}^{2^{j}-1} c_{j, k}\left(g_{1}(\cdot)\right) 2^{-(j+1)} .
\end{gathered}
$$

Теперь для всех $\nu \in \mathbb{Z} \backslash\{0\}$ сравним коэффициенты при $e^{i \nu x}$ в системе $(2.8),(2.9)$ и получим систему из двух уравнений

$$
\begin{gathered}
\sum_{j=\nabla_{\varepsilon}^{|\nu|}}^{+\infty} \sum_{k=0}^{2^{j}-1} c_{j, k}\left(\frac{\partial U_{1}}{\partial r}(1, \cdot)\right) 2^{-(j+1)} \widehat{\varphi}_{s}\left(\frac{\nu}{2^{j+1}}\right) e^{-2 \pi i \nu(2 k+1) / 2^{j+1}} \\
+\rho^{|\nu|} \sum_{j=\nabla_{\varepsilon}^{|\nu|}}^{+\infty} \sum_{k=0}^{2^{j}-1} c_{j, k}\left(\rho \frac{\partial U_{\rho}}{\partial r}(\rho, \cdot)\right) 2^{-(j+1)} \widehat{\varphi}_{s}\left(\frac{\nu}{2^{j+1}}\right) e^{-2 \pi i \nu(2 k+1) / 2^{j+1}} \\
=\sum_{j=\nabla_{\varepsilon}^{|\nu|}}^{+\infty} \sum_{k=0}^{2^{j}-1} c_{j, k}\left(g_{1}(\cdot)\right) 2^{-(j+1)} \widehat{\varphi}_{s}\left(\frac{\nu}{2^{j+1}}\right) e^{-2 \pi i \nu(2 k+1) / 2^{j+1}}
\end{gathered}
$$




$$
\begin{gathered}
-\rho^{|\nu|} \sum_{j=\nabla_{\varepsilon}^{|\nu|}}^{+\infty} \sum_{k=0}^{2^{j}-1} c_{j, k}\left(\frac{\partial U_{1}}{\partial r}(1, \cdot)\right) 2^{-(j+1)} \widehat{\varphi}_{s}\left(\frac{\nu}{2^{j+1}}\right) e^{-2 \pi i \nu(2 k+1) / 2^{j+1}} \\
-\sum_{j=\nabla_{\varepsilon}^{|\nu|}}^{+\infty} \sum_{k=0}^{2^{j}-1} c_{j, k}\left(\rho \frac{\partial U_{\rho}}{\partial r}(\rho, \cdot)\right) 2^{-(j+1)} \widehat{\varphi}_{s}\left(\frac{\nu}{2^{j+1}}\right) e^{-2 \pi i \nu(2 k+1) / 2^{j+1}} \\
=\rho \sum_{j=\nabla_{\varepsilon}^{|\nu|}}^{+\infty} \sum_{k=0}^{2^{j}-1} c_{j, k}\left(g_{\rho}(\cdot)\right) 2^{-(j+1)} \widehat{\varphi}_{s}\left(\frac{\nu}{2^{j+1}}\right) e^{-2 \pi i \nu(2 k+1) / 2^{j+1}}
\end{gathered}
$$

Решая данную систему из двух уравнений относительно не самих неизвестных коэффициентов $c_{j, k}$, а сумм по $j$ и $k$ с этими неизвестными коэффициентами и соответствующими множителями, получим при всех целых $\nu \neq 0$

$$
\begin{gathered}
\sum_{j=\nabla_{\varepsilon}^{|\nu|}}^{+\infty} \sum_{k=0}^{2^{j}-1} c_{j, k}\left(\frac{\partial U_{1}}{\partial r}(1, \cdot)\right) 2^{-(j+1)} \widehat{\varphi}_{s}\left(\frac{\nu}{2^{j+1}}\right) e^{-2 \pi i \nu(2 k+1) / 2^{j+1}} \\
=\frac{1}{1-\rho^{2|\nu|}} \sum_{j=\nabla_{\varepsilon}^{|\nu|}}^{+\infty} \sum_{k=0}^{2^{j}-1}\left(c_{j, k}\left(g_{1}(\cdot)\right)+\rho^{|\nu|+1} c_{j, k}\left(g_{\rho}(\cdot)\right)\right) 2^{-(j+1)} \widehat{\varphi}_{s}\left(\frac{\nu}{2^{j+1}}\right) e^{-2 \pi i \nu(2 k+1) / 2^{j+1}} \\
\\
\sum_{j=\nabla_{\varepsilon}^{|\nu|}}^{+\infty} \sum_{k=0}^{2^{j}-1} c_{j, k}\left(\rho \frac{\partial U_{\rho}}{\partial r}(\rho, \cdot)\right) 2^{-(j+1)} \widehat{\varphi}_{s}\left(\frac{\nu}{2^{j+1}}\right) e^{-2 \pi i \nu(2 k+1) / 2^{j+1}} \\
=-\frac{1}{1-\rho^{2|\nu|}} \sum_{j=\nabla_{\varepsilon}^{|\nu|}}^{+\infty} \sum_{k=0}^{2^{j}-1}\left(\rho^{|\nu|} c_{j, k}\left(g_{1}(\cdot)\right)+\rho c_{j, k}\left(g_{\rho}(\cdot)\right)\right) 2^{-(j+1)} \widehat{\varphi}_{s}\left(\frac{\nu}{2^{j+1}}\right) e^{-2 \pi i \nu(2 k+1) / 2^{j+1}}
\end{gathered}
$$

Таким образом, формулы из (2.7) примут вид

$$
\begin{gathered}
r \frac{\partial U_{1}}{\partial r}(r, x)=\frac{\partial U_{1}}{\partial r}(1,0)+\sum_{j=0}^{+\infty} \sum_{k=0}^{2^{j}-1} 2^{-(j+1)}\left(c_{j, k}\left(\frac{\partial U_{1}}{\partial r}(1, \cdot)\right)\right. \\
\left.+\left.\sum_{\nu \in \Delta_{\varepsilon}^{j+1} \cap \mathbb{Z} \backslash\{0\}}\left(c_{j, k}\left(g_{1}(\cdot)\right)+\rho^{|\nu|+1} c_{j, k}\left(g_{\rho}(\cdot)\right)\right) \widehat{\varphi}_{s}\left(\frac{\nu}{2^{j+1}}\right) \frac{1}{1-\rho^{2|\nu|}}\right|^{|\nu|} e^{i \nu\left(x-2 \pi(2 k+1) / 2^{j+1}\right)}\right), \\
(r, x): z=r e^{i x} \in \overline{K_{1}} ; \\
r \frac{\partial U_{\rho}}{\partial r}(r, x)=\rho \frac{\partial U_{\rho}}{\partial r}(\rho, 0)+\sum_{j=0}^{+\infty} \sum_{k=0}^{2^{j}-1} 2^{-(j+1)}\left(c_{j, k}\left(\rho \frac{\partial U_{\rho}}{\partial r}(\rho, \cdot)\right)\right. \\
-\sum_{\nu \in \Delta_{\varepsilon}^{j+1} \cap \mathbb{Z} \backslash\{0\}}\left(\rho^{|\nu|} c_{j, k}\left(g_{1}(\cdot)\right)+\rho c_{j, k}\left(g_{\rho}(\cdot)\right)\right) \widehat{\varphi}_{s}\left(\frac{\nu}{2^{j+1}}\right) \frac{1}{\left.1-\rho^{2|\nu|}\left(\frac{\rho}{r}\right)^{|\nu|} e^{i \nu\left(x-2 \pi(2 k+1) / 2^{j+1}\right)}\right),} \\
(r, x): z=r e^{i x} \in \mathbb{C} \backslash K_{\rho} .
\end{gathered}
$$

Теперь, имея из (2.5) представление

$$
r \frac{\partial U(r, x)}{\partial r}=r \frac{\partial U_{1}(r, x)}{\partial r}+r \frac{\partial U_{\rho}(r, x)}{\partial r}+A,
$$

с учетом только что полученных формул для коэффициентов разложения функций

$$
r \frac{\partial U_{1}}{\partial r} \text { и } r \frac{\partial U_{\rho}(r, x)}{\partial r}
$$


по гармоническим полиномам

$$
\Phi_{s}^{j+1,2 k+1}(r, x) \quad \text { и } \Phi_{s}^{j+1,2 k+1}(\rho / r, x)
$$

соответственно и найденного $A$ можно получить уже саму функцию $U(r, x)$, поделив на $r$ $(\rho<r<1)$ и проинтегрировав по $r$ обе части рассматриваемого равенства:

$$
\begin{gathered}
U(r, x)=\sum_{j=0}^{+\infty} \sum_{k=0}^{2^{j}-1} 2^{-(j+1)} \\
\times \sum_{\nu \in \Delta_{\varepsilon}^{j+1} \cap \mathbb{Z} \backslash\{0\}}\left(c_{j, k}\left(g_{1}(\cdot)\right)+\rho^{|\nu|+1} c_{j, k}\left(g_{\rho}(\cdot)\right)\right) \widehat{\varphi}_{s}\left(\frac{\nu}{2^{j+1}}\right) \frac{1}{|\nu|\left(1-\rho^{2|\nu|}\right)} r^{|\nu|} e^{i \nu\left(x-2 \pi(2 k+1) / 2^{j+1}\right)} \\
+\sum_{j=0}^{+\infty} \sum_{k=0}^{2^{j}-1} 2^{-(j+1)} \\
\sum_{\nu \in \Delta_{\varepsilon}^{j+1} \cap \mathbb{Z} \backslash\{0\}}\left(\rho^{|\nu|} c_{j, k}\left(g_{1}(\cdot)\right)+\rho c_{j, k}\left(g_{\rho}(\cdot)\right)\right) \widehat{\varphi}_{s}\left(\frac{\nu}{2^{j+1}}\right) \frac{1}{|\nu|\left(1-\rho^{2|\nu|}\right)}\left(\frac{\rho}{r}\right)^{|\nu|} e^{i \nu\left(x-2 \pi(2 k+1) / 2^{j+1}\right)} \\
+\left(g_{1}(0)+\sum_{j=0}^{+\infty} \sum_{k=0}^{2^{j}-1} c_{j, k}\left(g_{1}(\cdot)\right) 2^{-(j+1)}\right) \ln r+U_{0}, \quad(r, x): z=r e^{i x} \in \overline{R_{\rho}},
\end{gathered}
$$

где $U_{0}$ - произвольная вещественная константа. Сгруппируем оба только что полученных ряда относительно коэффициентов $c_{j, k}\left(g_{1}(\cdot)\right), c_{j, k}\left(g_{\rho}(\cdot)\right)$ и получим представление $(2.4)$. Так как найденная функция $U(r, x)$ гармоническая в $R_{\rho}$, то для того чтобы доказать равномерную сходимость в $\overline{R_{\rho}}$ обоих рядов из правой части $(2.4)$, достаточно доказать равномерную сходимость этих рядов на границе $R_{\rho}$. При $r=\rho$ оба ряда тождественно равны 0 . При $r=1$ оба ряда сходятся равномерно в силу признака Абеля.

Теорема доказана.

Найдем теперь оценку для погрешности аппроксимации точного решения задачи (2.1) частичными суммами порядка $J \in\{0\} \cup \mathbb{N}$ первого ряда из правой части формулы $(2.4)$ и второго ряда из правой части формулы $(2.4)$, обозначенными через $S_{s, 2^{J}}\left(r, x ; U, g_{1}\right)$ и $S_{s, 2^{J}}\left(r, x ; U, g_{\rho}\right)$ соответственно (константу $U_{0}$ можно без ограничения общности включить в частичную суммy $\left.S_{s, 2^{J}}\left(r, x ; U, g_{1}\right)\right)$.

Теорема 2. В условиях, сбормулированных при постановке задачи (2.1), получаем, что при каждом $s=1,2,3$ и $J \in\{0\} \cup \mathbb{N}$ частичная сумма $S_{s, 2^{J}}\left(r, x ; U, g_{1}, g_{\rho}\right)$ ряда из правой части (2.4) приближает точное решение $U(r, x)$ задачи (2.1) с оценкой

$$
\begin{gathered}
\left\|U(r, x)-\left(S_{s, 2^{J}}\left(r, x ; U, g_{1}\right)+S_{s, 2^{J}}\left(r, x ; U, g_{\rho}\right)\right)\right\|_{C\left(R_{\rho}\right)} \\
\leq \frac{\pi\left(1+\rho^{2}\right)}{\sqrt{3}(1-\rho)}\left(1+\left\|S_{s, 2^{J}}\right\|_{C_{2 \pi}}\right)\left(E_{N^{-}(\varepsilon, J)}\left(g_{1}\right)_{C_{2 \pi}}+E_{N^{-}(\varepsilon, J)}\left(g_{\rho}\right)_{C_{2 \pi}}\right),
\end{gathered}
$$

где $E_{N_{\varepsilon, J}}(f)_{C_{2 \pi}}$ - наилучшее приближение функиии $f$ в $C_{2 \pi}$ тригонометрическими полиномами порядка

$$
N_{\varepsilon, J}^{-}:=\left\lfloor 2^{J-1}(1-\varepsilon)\right\rfloor
$$

Оченки для нормы оператора $S_{s, 2}$ м можно найти в [4, теорема].

Д о к а з а т е л ь с т в о. Зафиксируем $J \in\{0\} \cup \mathbb{N}$. Используя доказательство утвержде- 
ния [6, теорема 2], можно получить следующие представления:

$$
\begin{aligned}
& U(r, x)=\frac{1}{\pi} \int_{0}^{2 \pi} \sum_{\mu=1}^{+\infty} \frac{\cos (\mu(x-\xi))}{\mu} \\
& \times\left(\sum_{j=0}^{+\infty} \sum_{k=0}^{2^{j}-1} 2^{-(j+1)} \sum_{\nu \in \Delta_{\varepsilon}^{j+1} \cap \mathbb{Z}} c_{j, k}\left(g_{1}(\cdot)\right) \widehat{\varphi}_{s}\left(\frac{\nu}{2^{j+1}}\right) \frac{r^{2|\nu|}+\rho^{2|\nu|}}{r^{|\nu|}\left(1-\rho^{2|\nu|}\right)} e^{i \nu\left(x-2 \pi(2 k+1) / 2^{j+1}\right.}\right) \\
& +\sum_{j=0}^{+\infty} \sum_{k=0}^{2^{j}-1} 2^{-(j+1)} \sum_{\nu \in \Delta_{\varepsilon}^{j+1} \cap \mathbb{Z}} c_{j, k}\left(g_{\rho}(\cdot)\right) \widehat{\varphi}_{s}\left(\frac{\nu}{2^{j+1}}\right) \frac{\rho^{|\nu|+1}\left(r^{2|\nu|}+1\right)}{r^{|\nu|}\left(1-\rho^{2|\nu|}\right)} e^{i \nu\left(x-2 \pi(2 k+1) / 2^{j+1}\right)} \\
& \left.+\left(g_{1}(0)+\sum_{j=0}^{+\infty} \sum_{k=0}^{2^{j}-1} c_{j, k}\left(g_{1}(\cdot)\right) 2^{-(j+1)}\right) \ln r+U_{0}\right) d \xi \\
& S_{s, 2^{J}}\left(r, x ; U, g_{1}\right)=\frac{1}{\pi} \int_{0}^{2 \pi} \sum_{\mu=1}^{+\infty} \frac{\cos (\mu(x-\xi))}{\mu} \\
& \times\left(\sum_{j=0}^{J-1} \sum_{k=0}^{2^{j}-1} 2^{-(j+1)} \sum_{\nu \in \Delta_{\varepsilon}^{j+1} \cap \mathbb{Z}}\left(c_{j, k}\left(g_{1}(\cdot)\right)+c_{j, k}\left(g_{\rho}(\cdot)\right)\right) \widehat{\varphi}_{s}\left(\frac{\nu}{2^{j+1}}\right) \frac{r^{2|\nu|}+\rho^{2|\nu|}}{r^{|\nu|}\left(1-\rho^{2|\nu|}\right)} e^{i \nu\left(x-2 \pi(2 k+1) / 2^{j+1}\right)}\right. \\
& \left.+\left(g_{1}(0)+\sum_{j=0}^{+\infty} \sum_{k=0}^{2^{j}-1} c_{j, k}\left(g_{1}(\cdot)\right) 2^{-(j+1)}\right) \ln r+U_{0}\right) g d \xi \\
& S_{s, 2^{J}}\left(r, x ; U, g_{\rho}\right)=\frac{1}{\pi} \int_{0}^{2 \pi} \sum_{\mu=1}^{+\infty} \frac{\cos (\mu(x-\xi))}{\mu} \\
& \times\left(\sum_{j=0}^{J-1} \sum_{k=0}^{2^{j}-1} 2^{-(j+1)} \sum_{\nu \in \Delta_{\varepsilon}^{j+1} \cap \mathbb{Z}}\left(c_{j, k}\left(g_{1}(\cdot)\right)+c_{j, k}\left(g_{\rho}(\cdot)\right)\right) \widehat{\varphi}_{s}\left(\frac{\nu}{2^{j+1}}\right) \frac{\rho^{|\nu|+1}\left(r^{2|\nu|}+1\right)}{r^{|\nu|}\left(1-\rho^{2|\nu|}\right)} e^{i \nu\left(x-2 \pi(2 k+1) / 2^{j+1}\right)}\right) d \xi .
\end{aligned}
$$

Обозначим через $S_{s, 2^{J}}\left(r, x ; g_{1}\right)$ и $S_{s, 2^{J}}\left(r, x ; g_{\rho}\right)$ частичные суммы рядов из первого и второго равенств (2.3) соответственно. Используя только что полученные представления и проводя выкладки, аналогичные выкладкам из доказательства утверждения [6, теорема 2], получим

$$
\begin{aligned}
& \leq\left|U(r, x)-\left(S_{s, 2^{J}}\left(r, x ; U, g_{1}\right)+S_{s, 2^{J}}\left(r, x ; U, g_{\rho}\right)\right)\right| \\
& \leq \quad\left(\mid \sum_{j=0}^{+\infty} \sum_{k=0}^{2^{j}-1} 2^{-(j+1)} \sum_{\nu \in \Delta_{\varepsilon}^{j+1} \cap \mathbb{Z} \backslash\{0\}} c_{j, k}\left(g_{1}(\cdot)\right) \widehat{\varphi}_{s}\left(\frac{\nu}{2^{j+1}}\right) \frac{1+\rho^{2|\nu|}}{1-\rho^{2|\nu|}} e^{i \nu\left(x-2 \pi(2 k+1) / 2^{j+1}\right)}\right. \\
& -\sum_{j=0}^{J-1} \sum_{k=0}^{2^{j}-1} 2^{-(j+1)} \sum_{\nu \in \Delta_{\varepsilon}^{j+1} \cap \mathbb{Z} \backslash\{0\}} c_{j, k}\left(g_{1}(\cdot)\right) \widehat{\varphi}_{s}\left(\frac{\nu}{2^{j+1}}\right) \frac{1+\rho^{2|\nu|}}{1-\rho^{2|\nu|}} e^{i \nu\left(x-2 \pi(2 k+1) / 2^{j+1}\right)} \mid \\
& +\mid \sum_{j=0}^{+\infty} \sum_{k=0}^{2^{j}-1} 2^{-(j+1)} \sum_{\nu \in \Delta_{\varepsilon}^{j+1} \cap \mathbb{Z} \backslash\{0\}} c_{j, k}\left(g_{\rho}(\cdot)\right) \widehat{\varphi}_{s}\left(\frac{\nu}{2^{j+1}}\right) \rho \frac{\rho^{2|\nu|}+1}{1-\rho^{2|\nu|}} e^{i \nu\left(x-2 \pi(2 k+1) / 2^{j+1}\right)} \\
& \left.-\sum_{j=0}^{J-1} \sum_{k=0}^{2^{j}-1} 2^{-(j+1)} \sum_{\nu \in \Delta_{\varepsilon}^{j+1} \cap \mathbb{Z} \backslash\{0\}} c_{j, k}\left(g_{\rho}(\cdot)\right) \widehat{\varphi}_{s}\left(\frac{\nu}{2^{j+1}}\right) \rho \frac{\rho^{2|\nu|}+1}{1-\rho^{2|\nu|}} e^{i \nu\left(x-2 \pi(2 k+1) / 2^{j+1}\right)} \mid\right)
\end{aligned}
$$




$$
\begin{aligned}
& \leq \frac{\pi\left(1+\rho^{2}\right)}{\sqrt{3}(1-\rho)}\left(\left|g_{1}(x)-S_{s, 2^{J}}\left(r, x ; g_{1}\right)\right|+\left|g_{\rho}(x)-S_{s, 2^{J}}\left(r, x ; g_{\rho}\right)\right|\right) \\
\leq & \frac{\pi\left(1+\rho^{2}\right)}{\sqrt{3}(1-\rho)}\left(1+\left\|S_{s, 2^{J}}\right\|_{C_{2 \pi}}\right)\left(E_{N^{-}(\varepsilon, J)}\left(g_{1}\right)_{C_{2 \pi}}+E_{N^{-}(\varepsilon, J)}\left(g_{\rho}\right)_{C_{2 \pi}}\right) .
\end{aligned}
$$

Теорема доказана.

\section{Заключение}

В статье получено решение задачи Неймана в центрально-симметричном кольце, представленное в виде двух рядов по гармоническим интерполяционно-ортогональным $2 \pi$-периодическим всплескам. Отметим достоинства данного решения. Полученные ряды равномерно сходятся в замыкании области (в нашем случае в замыкании кольца). Ряды Фурье, также применяемые для решения краевых задач, не обладают этим свойством. Коэффициенты полученных рядов требуют выполнения лишь простейших арифметических операций. Это значительно упрощает численную реализацию данного метода - не требуется вычислять интегралы, как в случае рядов Фурье или только ортогональных $2 \pi$-периодических всплесков. Также частичные суммы полученных рядов аппроксимируют точное решение задачи Неймана с порядком наилучшего приближения тригонометрическими полиномами.

Автор благодарит своего научного руководителя профессора Н. И. Черных за постановку задачи, обсуждение результатов и постоянное внимание к исследованиям автора.

\section{СПИСОК ЛИТЕРАТУРЫ}

1. Субботин Ю.Н., Черных Н. И. Гармонические всплески в краевых задачах для гармонических и бигармонических функций // Тр. Ин-та математики и механики УрО РАН. 2010. Т. 16, № 4. C. 281-296. doi: 10.1134/S0081543811050154 .

2. Meyer Y Ondelettes et opérateurs. Paris: Herman, 1990. 215 p.

3. Offin D., Oskolkov K. A note on orthonormal polynomial bases and wavelets // Constructive Approximation. 1993. Vol. 9. P. 319-325. doi: 10.1007/BF01198009.

4. Субботин Ю.Н., Черных Н. И. Интерполяционные всплески в краевых задачах // Тр. Ин-та математики и механики УрО РАН. 2016. Т. 22, № 4. С. 257-268. doi: 10.21538/0134-4889-2016-22-4-257-268 .

5. Субботин Ю.Н., Черных Н. И. Интерполяционно-ортогональные системы всплесков // Тр. Инта математики и механики УрО РАН. 2008. Т. 14, № 3. С. 153-161. doi: 10.1134/S0081543809050083.

6. Yamkovoi D. Harmonic interpolating wavelets in Neumann boundary value problem in a circle // Ural Math. J. 2019. Vol. 5, № 1. P. 91-100. doi: 10.15826/umj.2019.1.009.

7. Голузин Г.М. Решение основных плоских задач математической физики для случая уравнения Laplace'a и многосвязных областей, ограниченных окружностями (метод функциональных уравнений) // Мат. сб. 1934. Т. 41, № 2. С. 246-276.

Поступила 1.08.2020

После доработки 16.10.2020

Ямковой Дмитрий Анатольевич

Принята к публикации 23.10.2020

младший науч. сотрудник

Институт математики и механики им. Н.Н. Красовского УрО РАН;

ассистент

Уральский федеральный университет

г. Екатеринбург

e-mail: dmitriiyamkovoi@bk.ru 


\section{REFERENCES}

1. Subbotin Yu.N., Chernykh N.I. Harmonic wavelets in boundary value problems for harmonic and biharmonic functions. Proc. Steklov Inst. Math. (Suppl.), 2011, vol. 273, no. 1, pp. 142-159. doi: $10.1134 /$ S0081543811050154.

2. Meyer Y. Ondelettes et opérateurs. Paris: Herman, 1990, 215 p. ISBN: 2705661255.

3. Offin D., Oskolkov K. A note on orthonormal polynomial bases and wavelets. Constructive Approximation, 1993, vol. 9, pp. 319-325. doi: 10.1007/BF01198009.

4. Subbotin Yu.N., Chernykh N.I. Interpolation wavelets in boundary value problems. Proc. Steklov Inst. Math. (Suppl.), 2018, vol. 300, no. 1, pp. 172-183. doi: 10.1134/S0081543818020177.

5. Subbotin Yu.N., Chernykh N. I. Interpolating-orthogonal wavelet systems. Proc. Steklov Inst. Math. (Suppl.), 2009, vol. 264, no. 1, pp. 107-115. doi: 10.1134/S0081543809050083 .

6. Yamkovoi D. Harmonic interpolating wavelets in Neumann boundary value problem in a circle. Ural Math. J., 2019, vol. 5, no. 1, pp. 91-100. doi: 10.15826/umj.2019.1.009 .

7. Goluzin G.M. The solution of the basic plane problems of a mathematical physics for the case of Laplace equations and a multiply connected domains bounded by circular curves (the method of functional equations). Sbornik: Mathematics, 1934, vol. 41, no. 2, pp. 246-276 (in Russian).

Received August 1, 2020

Revised October 16, 2020

Accepted October 23, 2020

Funding Agency: This study is a part of the research carried out at the Ural Mathematical Center.

Dmitry Anatolyevich Yamkovoi, Krasovskii Institute of Mathematics and Mechanics of the Ural Branch of the Russian Academy of Sciences, Yekaterinburg, 620108 Russia; Ural Federal University, Yekaterinburg, 620000 Russia, e-mail: dmitriiyamkovoi@bk.ru .

Cite this article as: D. A. Yamkovoi. Harmonic interpolating wavelets in the Neumann boundary value problem in a ring, Trudy Instituta Matematiki i Mekhaniki UrO RAN, 2020, vol. 26, no. 4, pp. 279-289 . 